\title{
The attentional demands of mnemonic control processes
}

\author{
DOUGLAS GRIFFITH \\ University of Utah, Salt Lake City, Utah 84112
}

\begin{abstract}
In order to assess the processing (i.e., attentional) demands of different control processes, subjects were required to perform a secondary choice reaction time task in addition to primary verbal tasks. The performance of the secondary task yielded a measure termed expended processing capacity (EPC), which was used to infer the attentional demands of the primary tasks. Two factors, mediator type (experimenter-supplied vs. subject-generated) and intentionality (incidental vs. intentional), were varied in a paired-associate situation in an effort to affect the degree of elaboration processing. Only mediator type had an effect on recall accuracy and on EPC during both initial processing and recall. Subject-generated mediators resulted in higher recall and in higher EPC during initial processing, but in lower EPC during recall, than did experimenter-supplied mediators. Implications of these findings are discussed.
\end{abstract}

Given the number and variety of mnemonic control processes that have been proposed, it appears necessary to develop empirical techniques to distinguish among them. At a theoretical level, Craik and Lockhart (1972) have distinguished two general classes of control processes: Type I, or simple maintenance processes, in which information is merely maintained at some level in the processing system, and Type II, or elaboration processes, in which information is processed to a greater depth. Here "depth" refers to the degree of semantic or cognitive analysis. Apparently this theoretical distinction has significant empirical consequences, since certain studies indicate that only when Type II processing is employed will retention vary as a function of processing time (Craik \& Watkins, 1973; Modigliani \& Seamon, 1974). Moreover, it appears that, whereas Type I processing might be advantageous for the immediate recall of information, Type II processing results in superior long-term retention (Watkins \& Watkins, 1974).

Since the assumed differences between Type I and Type II processes seem to have such pronounced effects on recall, it would be useful if these classes of processing could be distinguished during the initial input of information. Assuming an "effort" concept of attention similar to that advocated by Kahneman (1973), a likely candidate would be the attention, or mental effort, required to implement a control process. It may be that Type II processes require greater mental effort to implement. If such is the case, then an attentional

This report is based on part of a dissertation submitted to the University of Utah in partial fulfillment of the requirements of the PhD degree. The research was conducted while the author was supported by a National Institute of Mental Health Traineeship. The author would like to thank his committee chairman, William A. Johnston, and the committee members, C. N. Uhl, W. F. Prokasy, D. H. Dodd, P. B. Porter, and $R$. Kesner, for their helpful comments. The author is now at the Army Research Institute Field Unit, HQ MASSTER, Fort Hood, Texas 76544. dimension would be of value in distinguishing these two general classes of control processes.

Clearly, what is needed is a metric of mental effort or attention. Johnston and his colleagues have developed a technique which appears to supply such a metric (see Griffith \& Johnston, 1973; Johnston, Griffith, \& Wagstaff, 1972; Johnston, Wagstaff, \& Griffith, 1972). Based on a divided attention methodology, the technique requires the subject to perform a secondary task (e.g., a simple or choice reaction time task) in addition to the primary task of interest. Since the subject has only a finite capacity to process information, secondary task performance should suffer as the processing demands of the primary task increase. Thus secondary task performance can be used to make inferences about the processing demands of the primary task. That is, the greater the processing demands of the primary task, the poorer the performance of the secondary task, and the greater the inferred expended processing capacity (EPC).

The research strategy, then, is to assess EPC while manipulating classes of control processes. Since EPC should vary as a function of a variety of factors, it is critical that the experimental conditions be as comparable as possible so that the attentional demands of elaboration vs. maintenance processes can be assessed accurately. Ideally, control processes entailing the same basic mode of processing but varying in terms of the degree of elaboration required should be compared. With respect to the paired-associate situation, Bobrow and Bower (1969) have found, using an incidental learning paradigm, that when the mediator between two nouns is generated by the subject (subject-generated) recall is much better than when the mediator is supplied by the experimenter (experimenter-supplied). Since the processing in the case of the subject-generated mediators involves elaboration while the processing in the case of the experimenter-supplied mediators merely involves the 
short-term maintenance of information, it can be said that the latter (experimenter-supplied) is an instance of Type I processing primarily, whereas the former (subject-generated) is an instance of Type II processing primarily. Moreover, the same basic mode of processing, i.e., verbal mediation, is employed in both cases. Varying mediator type, then, seems to provide an ideal means of assessing the attentional demands of two general classes of control processes.

A second factor, which on intuitive grounds might be expected to influence the degree of elaboration processing, is intentionality. That is, subjects who anticipate a recall test might elaborate their processing in order to enhance their recall. If subjects do elaborate their processing, then it should be reflected in terms of higher EPC. Moreover, it is likely that the effect of intentionality is localized to experimentersupplied pairs, since elaboration processing is assumed to be implicit to subject-generated pairs. If such is the case, then the effect of intentionality on EPC during initial list processing as well as on recall accuracy should be greater for experimenter-supplied than for subjectgenerated pairs.

An ancillary result of some interest concerns the attentional demands during retrieval. That is, given that Type II processing results in superior long-term retention, does the actual retrieval of this information require less processing capacity than the retrieval of information initially processed under a Type I processing set? Since it is difficult to assess what is happening when a subject either fails to recall an item or recalls an item incorrectly, the EPC assessments of primary interest are those measures associated with items which are correctly recalled. That is, given correct recall, do items initially processed under a Type II processing set result in lower EPC during recall than items initially processed under a Type I processing set?

In order to assess EPC, the present study employed a choice reaction time (CRT) task as the secondary task. Subjects were required to press a "yes" button whenever they heard a digit from a designated set of four and a "no" button otherwise. In accordance with the mensural logic for EPC, subjects were instructed to devote the majority of their attention to the verbal processing tasks and any remaining attention to the CRT task.

The present study, then, compared the processing demands of experimenter-supplied and subject-generated mediators for noun paired associates during both initial list processing and recall by having subjects perform a concurrent CRT task. The effect of intentionality on recall and EPC was also assessed by informing half the subjects of the impending recall test. It was expected that recall for subject-generated pairs, those entailing Type II processing primarily, would be significantly higher than recall for experimenter-supplied pairs, those entailing Type I processing primarily. The primary purpose of this experiment, however, was to see if recall differences would be reflected in the EPC measure during initial list processing. Such a result would suggest the future utility of assessing control processes in terms of this attentional measure.

\section{METHOD}

\section{Tasks and Apparatus}

Four basic tasks were performed by each subject: a CRT task, a sentence repetition task (in the case of experimentersupplied mediators), a sentence generation task (in the case of subject-generated mediators), and a recall task. In order to monitor EPC, the CRT task was performed concurrently with each of the other tasks, so a derived fifth task was a divided attention task. The temporal sequencing of events on a trial for the four fundamental tasks adhered to the same basic pattern and is illustrated in Figure 1. No intertrial interval was employed; the following trial commenced as soon as the preceding one had finished. In order to aid the subject in discriminating between the tasks, all the CRT stimuli were presented over one channel of the headset, while all the other information was presented over the second channel.

The CRT task required the subject to respond by pressing a designated button whenever he heard a monosyllabic digit (one through nine excluding seven) over his headset. Four digits comprised the positive set to which the subject was to respond by pressing a button marked "YES." To the remaining four digits he was to respond by pressing a button marked "NO." The same digits comprised the positive and negative sets throughout the experimental session. Subjects were instructed to keep one finger on the YES button, which was always the button to the subject's right, and one finger on the NO button and to press the appropriate button as soon as they had classified the digit. While the instructions emphasized speed, they also emphasized the importance of errorless performance. A digit was presented within $4 \mathrm{sec}$ after the subject heard the preparatory "READY START." This digit activated the millisecond timer which was stopped by the subject's buttonpress. On the 8 th sec of the processing interval, a tone was presented. This procedure was followed to maintain the same temporal sequence as was employed in the divided attention trials (see below). A CRT trial was initiated every $10 \mathrm{sec}$

For the sentence repetition task, a sentence of the form "article Noun 1 verbed article Noun 2" (e.g., the STORM swept the HAMLET) was presented during the first $2 \mathrm{sec}$ of the processing interval. On the 8 th sec a tone was presented indicating that the subject was to repeat the sentence as quickly as possible so that he would be ready to hear the next sentence. The next sentence followed immediately after the $3 \mathrm{sec}$ allowed for sentence repetition.

The sentence generation task was structured the same way except that the two nouns were presented without the sentence context (e.g., STORM HAMLET). When the tone occurred, the subject was to repeat the sentence he had generated during the silent processing interval as quickly as possible so that he would be ready to hear the next pair of nouns. Subjects were required to withhold responding until the tone occurred to equate processing time between the mediation conditions.

During the recall phase, the subject heard a preparatory "RECALL" before each of the first words of the noun pairs was presented over one channel of the headset (e.g., RECALL STORM). On the 8th sec of the processing interval, the tone occurred indicating that the subject was to respond overtly if he thought he had recalled the item. This procedure was followed during the recall phase in order to maintain the same temporal patterning throughout the experiment.

On divided attention trials subjects were instructed to devote whatever attention they needed to the performance of the verbal tasks (i.e., sentence repetition, sentence generation, and recall tasks) and any remaining attention to the performance of the CRT task, with the restriction that they never completely 
Presentation Sequence During Baseline CRT

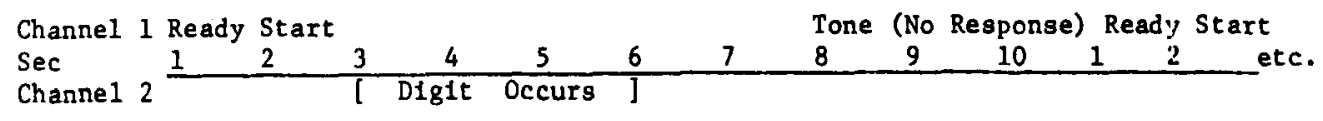

Presentation Sequence During Inftial L1st Processing (experimenter-supplied)

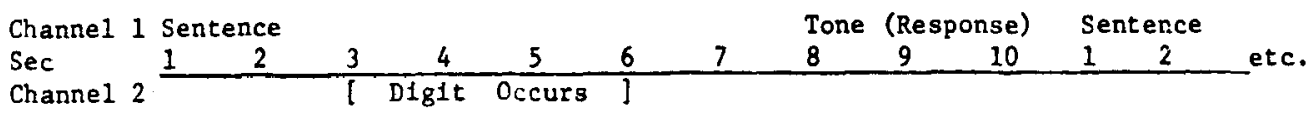

Presentation Sequence During Initial L1st Processing (subject-generated)

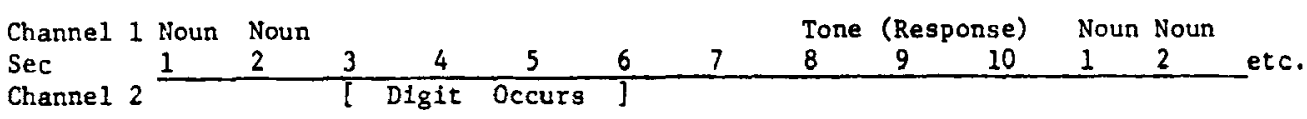

Presentation Sequence During Recall

\begin{tabular}{llllllllllll} 
Channel 1 Recall Noun & \multicolumn{1}{c}{ Tone (Response) Recall Noun } \\
Sec & 1 & 2 & 3 & 4 & 5 & 6 & 7 & 8 & 9 & 10 & 1
\end{tabular}

ignore the CRT task. The digit was always presented during the first $4 \mathrm{sec}$ of the silent processing interval in order to assure that the subjects could complete the CRT task prior to the response execution phase of the verbal task.

\section{Procedure}

The entire session was recorded on tape and played to the subjects on separate channels of a stereophonic headset. The digits were played over one channel of the headset, while all the other information was played over the second channel. Subjects were tested individually for one 40-min session in an experimental booth in which the experimenter and the subject were separated by an occluding partition. Taped instructions indicated that the experimenters were interested in the basic processes underlying the perception and generation of sentences as well as in how people distributed their attention between tasks. After receiving instructions about the sentence repetition and sentence generation tasks, subjects performed a practice list in which they first repeated sentences for eight pairs and then generated sentences for eight pairs. Subjects next received instructions explaining the CRT task followed by eight CRT practice trials. The subjects were then instructed as to how they were to perform the tasks concurrently. The practice phase was concluded with 16 practice trials in which they performed the verbal and CRT tasks concurrently (eight sentence rrepetition plus eight sentence generation trials). No recall tests were administered during the practice phase.

At the conclusion of the practice phase, subjects were informed that the experiment proper was to begin. First the subjects performed the CRT task by itself for 16 trials. Next the subjects were informed that they were to perform the two tasks concurrently and the instructions as to how they were to allocate their attention were reiterated. Following these instructions, the intentional subjects were forewarned of the impending recall test. Depending on the experimental conditions (see below), subjects were informed that they were either to generate or to repeat sentences. Thirty-two noun pairs
Fig. 1

followed, being presented in the context of sentences for the repetition task. Instructions then followed informing subjects who had repeated sentences that they were to generate sentences and informing subjects who had generated sentences that they were to repeat sentences. After the final 32 pairs had been presented, all subjects received identical recall instructions. When the subjects had been tested on all 64 pairs, the session was concluded with 16 CRT-only trials. At the end of the session, incidental subjects were administered a questionnaire assessing whether they had anticipated a retention test.

\section{Lists and Signal Programming}

Two initial 32-pair lists (A and B) of high-imagery nouns (all I values $>4.00$ ) were constructed from the Paivio, Yuille, and Madigan norms (1968). Sentences of the form "article Noun 1 verbed article Noun 2" were constructed for all noun pairs. Four lists of 32 pairs each were derived from each of these initial lists: two in which the noun pairs were presented in the context of sentences (Lists $A E$ and $B E$ ) and two in which the noun pairs were presented alone (Lists AS and BS). Four experimental lists were developed from these four basic lists by combining the basic lists such that the presentation order of the basic lists was counterbalanced (i.e., List AEBS, List BSAE, List ASBE, and List BEAS). One recall list was constructed such that all 64 noun pairs were tested in random order.

For the CRT task the digits were programmed to occur during the first $4 \mathrm{sec}$ of the silent processing interval. For each list the programming of these digits was random, with the restrictions that the digits occur equally often at each of the four signal loci and that there be an equal number of positive and negative instances at each of these loci. In programming the digits on the recall test, the above restrictions applied equally to experimenter-supplied and subjectgenerated pairs. The programming for the CRT-only list was done with the same restrictions, with the first 16 and second 16 trials regarded as one 32 -item list. 
Table 1

Summary of Means of Three Main Analyses

\begin{tabular}{|c|c|c|c|c|c|c|c|c|c|}
\hline \multirow[b]{2}{*}{ Mediator Type } & \multicolumn{3}{|c|}{ Recall Accuracy* } & \multicolumn{3}{|c|}{ Initial EPC** } & \multicolumn{3}{|c|}{ Recall EPC $\uparrow$} \\
\hline & Incidental & Intentional & Mean & Incidental & Intentional & Mean & Incidental & Intentional & Mean \\
\hline $\begin{array}{l}\text { Experimenter-Supplied } \\
\text { Subject-Generated }\end{array}$ & $\begin{array}{r}7.70 \\
12.85\end{array}$ & $\begin{array}{r}8.25 \\
14.10\end{array}$ & $\begin{array}{r}7.97 \\
13.47\end{array}$ & $\begin{array}{r}888 \\
1144\end{array}$ & $\begin{array}{r}945 \\
1150\end{array}$ & $\begin{array}{r}917 \\
1147\end{array}$ & $\begin{array}{l}1433 \\
1246\end{array}$ & $\begin{array}{l}1340 \\
1121\end{array}$ & $\begin{array}{l}1386 \\
1183\end{array}$ \\
\hline Mean & 10.27 & 11.17 & & 1016 & 1047 & & 1339 & 1230 & \\
\hline
\end{tabular}

*The entries represent the mean number of words correctly recalled (max $=32$ ).

**The entries are the means in milliseconds of the mean CRT for each subject equally weighted for positive and negative instances.

tThe entries are the means in milliseconds of the mean CRT contingent on correct recall for each subject equally weighted for positive and negative instances.

\section{Design and Subjects}

A basic 2 (instructional set) by 2 (mediator type) factorial design was employed where the two levels of instructional set were incidental vs. intentional learning and the two levels of mediator type were experimenter-supplied vs. subjectgenerated mediators. Repeated measures were taken on the second factor and list presentation conditions were counterbalanced within this basic design. Forty subjects were drawn from introductory and upper division psychology courses and were randomly but evenly assigned to each instructional set. Three incidental subjects had to be replaced for indicating that they had anticipated a retention test.

\section{RESULTS}

\section{Recall Accuracy}

An examination of Table 1 indicates that only mediator type had an effect on recall. As was expected, mean recall for subject-generated pairs was higher than for experimenter-supplied pairs. The analysis of variance of the total words correctly recalled, in which the factors were intentionality (incidental vs. intentional) and mediator type (experimenter-supplied vs. subjectgenerated), revealed the effect of mediator type, $\mathrm{F}(1,38)=61.71, \mathrm{p}<.001, \mathrm{MS}_{\mathrm{e}}=980.37$. Apparently the Type II processing assumed to be engendered by the sentence generation task enhanced information accessibility more than the Type I processing assumed to be engendered by the sentence repetition task. Neither the effect of intentionality nor the Intentionality by Mediator Type interaction was significant (both Fs < 1.00).

In retrospect, the failure of intentionality to affect the degree of elaboration processing is not particularly surprising. It may have been that the task demands for the experimenter-supplied pairs were such that they did not allow more extensive processing. The requirement to repeat sentences may have precluded further processing. Alternatively, since there are data suggesting that subjects do not modify their processing strategies until they receive feedback from a test trial (Paivio \& Yuille, 1969) and since there was only one recall trial, intentional subjects simply might not have been aware of the necessity to elaborate their processing in the case of the experimenter-supplied pairs.

\section{CRT Baseline}

The overall error rate was quite low, with subjects making only $1.8 \%$ errors in the incidental and $2.1 \%$ in the intentional conditions out of the 32 CRT-only trials. The mean of the correct CRTs equally weighted for positive and negative responses was determined for each subject, and these averaged $853 \mathrm{msec}$ and $873 \mathrm{msec}$ for the intentional and incidental conditions, respectively. A t test indicated that no correction for baseline performance was needed, $\mathrm{t}(38)=.06$.

\section{EPC During Initial List Processing}

The data confirmed initial expectations. The EPC was substantially higher for subject-generated than for experimenter-supplied pairs (see Table 1). An analysis of variance was conducted on the mean correct CRTs (maximum $n=32$ ) equally weighted for positive and negative instances. The factors were intentionality (incidental vs. intentional) and mediator type (experimenter-supplied vs. subject-generated). The test revealed the highly significant effect of mediator type, $\mathrm{F}(1,38)=76.19, \mathrm{p}<.001, \mathrm{MS}_{\mathrm{e}}=139,551.19$. Neither the effect of intentionality nor the interaction of Intentionality by Mediator Type was significant (both Fs $<1.00$ ). Since the subjects' performance of the two mediation tasks was quite high in an absolute sense, it can be safely inferred that the EPC measures are representative of successful task performance. Intentional and incidental subjects successfully generated sentences on $95 \%$ and $93 \%$ of the pairs, respectively, while both groups repeated the experimenter-supplied pairs correctly on $98 \%$ of the pairs. The performance of the CRT task was also quite high. Error rates were $1.8 \%, 2.4 \%, .9 \%$, and $2.7 \%$ for the intentional experimenter-supplied, intentional subjectgenerated, incidental experimenter-supplied, and incidental subject-generated conditions, respectively. What is most significant about the EPC data is their correspondence to the verbal recall data. In both analyses only the effect of mediator type was significant. Although subject-generated mediation required greater processing capacity to implement than experimenter-supplied mediation, the subject-generated 
mediation resulted in recall performance superior to that engendered by experimenter-supplied mediation.

\section{EPC During Recall}

When only mean correct CRTs contingent on correct recall were considered, it was found that recall of subject-generated pairs required less processing capacity than the recall of experimenter-supplied pairs (see Table 1). An analysis of variance of these data, again employing the factors of intentionality (incidental vs. intentional) and mediator type (experimenter-supplied vs. subject-generated), and again equally weighting CRTs for positive and negative responses, revealed a main effect of mediator type, $F(1,38)=12.55$, $\mathrm{p}<.01 \mathrm{MS}_{\mathrm{e}}=655,337.57$. Just as in the other analyses, neither the main effect of intentionality nor the interaction of Intentionality by Mediator Type was significant (both $F s<1.02$ ). Although the performance of the CRT task during recall was poorer than in the other task phases, an acceptable level was maintained. Subjects made $7.8 \%, 7.6 \%, 4.9 \%$, and $7.0 \%$ classification errors in the intentional experimentersupplied, intentional subject-generated, incidental experimenter-supplied, and incidental subject-generated conditions, respectively. Although caution should be observed in interpreting this analysis, since subjectitem selection artifacts present a problem with any conditional analysis, it appears that Type II processing resulted not only in a higher absolute level of recall, but also in a retrieval process which required less processing capacity to implement.

\section{DISCUSSION}

The EPC measure does appear useful in distinguishing maintenance (Type I) and elaboration (Type II) control processes. This distinction was quite pronounced during initial list processing where the elaboration process evidenced higher EPC than the maintenance process. Moreover, it appears that the type of processing employed during the initial processing of information affects not only the probability that the information will be retrieved, but also the amount of processing capacity required to retrieve the information. These results argue for the utility of an attentional measure in the experimental analysis of control processes.

Of course, the current study examined only the processing demands of experimenter-supplied and subject-generated mediators. Whether this is a general finding applicable to other maintenance and elaboration processes is a problem which requires further investigation. If this finding is a general one, however, it would suggest that Kahneman's (1973) effort notion of attention provides a useful conceptual framework for the study of mnemonic processes. Elaboration processes might require greater mental effort to implement, but they might also result in an enhancement of information accessibility. This enhanced accessibility would result in a retrieval process which requires relatively little processing capacity, which in turn results in higher recall. If this is the general case, that elaboration processes require greater mental effort than maintenance processes, it would suggest why subjects are not prone to elaborate information unless it is implicitly or explicitly demanded of them. It would also suggest the necessity for selection in information storage. If elaboration processes require additional capacity to implement, and if the subject has only a finite capacity to process information, then subjects must deploy their attentional capacity selectively, such that high-priority information is stored at the expense of information with a lower priority.

The relationship between attention and memory is not a simple one. It may be that the expenditure of additional processing capacity is a necessary, but not a sufficient, condition to enhance retention. Walsh and Jenkins (1973) have explicitly rejected an effort interpretation as the basis of mnemonic enhancement in favor of a process explanation. That the particular kind of processing employed is critical for retention is not a point of contention here. Clearly, a task can require a substantial amount of processing capacity, yet not result in a concomitant increase in retention. Thus, processing strategies need also to be evaluated along a qualitative dimension. Craik and Lockhart (1972) were careful to note that different processing strategies are differentially effective for different retention tests. The issue of the relative appropriateness of different processing strategies for different processing tasks represents a challenging problem that has recently been addressed by a number of investigators (e.g., Griffith, 1975; Jacoby, 1973). The present study suggests, however, that given an appropriate processing strategy, there is a positive relationship between processing capacity expended and recall.

Schwartz and Walsh (1974) have demonstrated that when the situation is contrived such that the mediators in the experimenter-supplied condition are identical to the mediators in the subject-generated condition, the recall difference between the conditions is eliminated. Unfortunately, Schwartz and Walsh did not assess EPC. According to the effort notion advanced in this paper, no differences in EPC during either the initial processing or the words or in their subsequent recall should have been obtained between the two conditions.

\section{REFERENCES}

Bobrow, S. A., \& Bower, G. H. Comprehension and recall of sentences. Joumal of Experimental Psychology, 1969, 80, 455-461.

CRAIK, F. I. M., \& LOCKharT, R. S. Levels of processing: A framework for memory research. Journal of Verbal Leaming and Verbal Behavior, 1972, 11, 671-684.

Craik, F. I. M., \& Watkins. M. J. The role of rehearsal in short-term memory. Journal of Verbal Learning and Verbal Behavior. 1973, 12, 599-607.

GRIfFITH. D. A comparison of control processes for recognition 
and recall. Journal of Experimental Psychology: Human Learning and Memory, 1975, 104, 223-228.

Griffith, D., \& Johnston, W. A. An information processing analysis of visual imagery. Journal of Experimental Psychology, 1973, 10), 141-146.

$\mathrm{J}_{\mathrm{ACOBY}}$. L. L. Test appropriate strategies in retention of categorized lists. Joumal of Verbal Learning and Verbal Behavior, 1973, 12, 675-682.

Johnston, W. A., Griffith, D., \& Wagstaff, R. R. Speed, accuracy, and ease of recall. Journal of Verbal Learning and Verbal Behavior, 1972, 11, 512-520.

Johnston. W. A., Wagstaff, R. R., \& Griffith, D. Information-processing analysis of verbal learning. Journal of Experimental Psychology, 1972, 96, 307-314.

Kahneman, D. Atention and effort. Englewood Cliffs, N.J: Prentice-Hall, 1973.

Modigliani. V., \& Seamon, J. G. Transfer of information from short- to long-term memory. Journal of Experimental Psychology, 1974, 102. 768-772.

Paivio, A., \& Yuille, J. C. Changes in associative strategies and paired associate learning over trials as a function of word imagery and type of learning set. Journal of Experimental
Psychology, 1969, 79, 458-463.

Paivio, A., Yuille, J. C., \& Madigan, S. Concreteness, imagery, and meaningfulness for 925 nouns. Journal of Experimental Psychology Monograph Supplement, 1968, 76(1, Part 2).

Schwartz, M., \& Walsh, M. F. Identical subject-generated and experimenter-supplied mediators in paired-associate learning. Joumal of Experimental Psychology, 1974, 103, 878-884.

WALSH, D. A., \& Jenkins, J. J. Effects of orienting tasks on free recall in incidental learning: "difficulty," "effort," and "process" explanations. Journal of Verbal Learning and Verbal Behavior, 1973, 12, 481-488.

Watkins, M. J., \& Watkins, O. C. Processing of recency items for free recall. Journal of Experimental Psychology, $1974,102,488-493$.

(Received for publication January 31, 1975; revision received May 9, 1975.) 